\title{
Potential vectors of Xylella fastidiosa: a study of leafhoppers and treehoppers in citrus agroecosystems affected by Citrus Variegated Chlorosis
}

\author{
G. Dellapé ${ }^{1,2 *}$, S. Paradell ${ }^{1}$, L. Semorile ${ }^{3} \&$ L. Delfederico ${ }^{3}$ \\ ${ }^{1}$ División Entomología, Museo de La Plata, Universidad Nacional de La Plata, Paseo del Bosque s/n, La Plata B1900FWA, \\ Argentina, ${ }^{2}$ Consejo Nacional de Investigaciones Científicas y Técnicas (CONICET), Buenos Aires, Argentina, and \\ ${ }^{3}$ Laboratorio de Microbiología Molecular, Universidad Nacional de Quilmes, Roque Saenz Peña 352, Bernal 1876, \\ Argentina \\ Accepted: 28 April 2016
}

Key words: Auchenorrhyncha, Hemiptera, Cicadellidae, Membracidae, sweet orange, tangerine, PCR, real-time PCR, molecular methods, seasonal fluctuation, Argentina

\section{Abstract}

\begin{abstract}
This study investigated the predominant leafhopper and treehopper (Hemiptera, Auchenorrhyncha) species in Citrus Variegated Chlorosis (CVC)-affected citrus agroecosystems in Argentina, their seasonal fluctuation, and their potential role as vectors of Xylella fastidiosa Wells et al., using molecular methods for detection. More than 6000 Auchenorrhyncha were collected from three citrus agroecosystems over a period of 3 years using yellow sticky traps and entomological nets. Cicadellidae and Membracidae were the most abundant families. Of the 43 species identified, five were predominant in citrus orchards, and three were predominant in weeds surrounding citrus plants. All predominant species and another four non-predominant species tested positive for X. fastidiosa in PCR and real-time PCR assays. In a transmission assay, Dechacona missionum (Berg), Tapajosa rubromarginata (Signoret), and Cyphonia clavigera (Fabricius) transmitted X. fastidiosa successfully. Scaphytopius bolivianus Oman and Frequenamia spiniventris (Linnavuori) populations increased once (during the summer), possibly due to favorable weather conditions, and Bucephalogonia xanthophis (Berg), Molomea lineiceps Young, and T. rubromarginata populations increased twice a year: once in summer and once in winter, coinciding with the increase in early citrus shoots (flush). Among the $X$. fastidiosa-positive species, those with the higher population densities during the sprouting period, where trees are highly susceptible to infection, must be considered as most relevant vectors of CVC in the citrus-growing areas in Argentina.
\end{abstract}

\section{Introduction}

Among the Hemiptera, the suborder Auchenorrhyncha represents a diverse group of exclusively phytophagous insects with recognized phytosanitary importance, as many species can be abundant and cause considerable damage to crops by feeding directly on the plants, or by acting as vectors of plant pathogens (Nielson, 1968; Nault \& Ammar, 1989; Álvarez et al., 2011). Xylella fastidiosa Wells et al. is a xylem-limited bacterial plant pathogen known to be transmitted by about 50 species of Auchenorrhyncha belonging to the families Cercopidae

*Correspondence: Gimena Dellapé, División Entomología, Museo de La Plata, Paseo del Bosque s/n, La Plata B1900FWA, Argentina.

E-mail: gimenadellape@gmail.com (froghoppers), Aphrophoridae (spittlebugs), Cicadellidae (leafhoppers), and Membracidae (treehoppers) (de Coll et al., 2000; Redak et al., 2004; Yamamoto et al., 2007; Zhang et al., 2011; Saponari et al., 2014). The members of Cicadidae and Tibicinidae are considered potential vectors (EFSA, 2015). The pathogen multiplies in the precibarium and cibarium of the vector and is apparently limited to this area of the foregut (Hill \& Purcell, 1995; Almeida \& Purcell, 2006); nymphs lose infectivity after molting when the cuticular lining of the foregut is shed (Purcell \& Finlay, 1979), and there is no evidence for transovarial transmission (Freitag, 1951; Janse \& Obradovic, 2010).

Four subspecies of $X$. fastidiosa have been genetically and biologically characterized (Chen et al., 1992; Pooler \& Hartung, 1995; da Costa et al., 2000; Almeida \& Purcell, 2003). In North America, the subspecies X. fastidiosa 
multiplex, X. fastidiosa fastidiosa, and X. fastidiosa sandyi cause economically important agricultural diseases, e.g., Pierce's disease in grapevines, Almond Leaf Scorch, and Oleander Leaf Scorch, whereas in South America, the subspecies X. fastidiosa pauca causes Coffee Leaf Scorch, Olive Scorch, and Citrus Variegated Chlorosis (CVC) (Li et al., 2001; Qin et al., 2001; Schaad et al., 2004; Schuenzel et al., 2005; Haelterman et al., 2015). Recently, X. fastidiosa pauca has also been reported in Europe as an emerging threat to olive trees, almond, oleander, and some other weed and ornamental hosts (Saponari et al., 2013, 2014; Loconsole et al., 2014), and X. fastidiosa multiplex has been reported in Polygala myrtifolia L. in France (EPPO, 2015).

In Argentina, CVC was first detected in the Misiones Province, from where it spread to the south, affecting citrus orchards in Corrientes and Entre Ríos Provinces (de Coll et al., 2000; Beltrán et al., 2004; Costa et al., 2009), and to the north, damaging citrus orchards in Brazil (Hopkins \& Purcell, 2002). Twelve leafhopper species are known to be vectors of CVC in Brazil (Lopes, 1996; Gravena et al., 1998; Parra et al., 2003; Fundecitrus, 2007). In Argentina, there is little information on the leafhoppers and treehoppers that could act as vectors of $X$. fastidiosa in CVC-affected citrus agroecosystems. The only studies on the subject were conducted by de Coll et al. $(1993,2000)$, who detected X. fastidiosa in leafhopper species from citrus in the Misiones Province using serological dot immunobinding assay (DIBA) techniques.

The design of efficient vector-insect management strategies requires prior knowledge of all the species present in CVC-affected citrus agroecosystems and their ability to act as vectors of $X$. fastidiosa. The aims of this study were to (1) identify the predominant leafhopper and treehopper species in three CVC-affected citrus agroecosystems in the main citrus-growing area in Argentina, (2) study the seasonal fluctuation of predominant species in citrus orchards, (3) detect the bacterium $X$. fastidiosa in leafhoppers and treehoppers using molecular methods, and (4) carry out a transmission assay to identify the possible vectors of a disease which is threatening the local citrus industry.

\section{Materials and methods}

\section{Study site}

Sampling was conducted at the INTA Agricultural Experimental Station $\left(31^{\circ} 22^{\prime} 28^{\prime \prime} \mathrm{S}, 58^{\circ} 06^{\prime} 59^{\prime \prime} \mathrm{W}, 45 \mathrm{~m}\right.$ a.s.l.) located in the Concordia Department, Entre Ríos Province, Argentina. Three CVC-affected citrus orchards were selected for this study: (Site 1) sweet orange variety 'Valencia Late', Citrus sinensis (L.) Osbeck, grafted onto trifoliate orange, Poncirus trifoliate (L.) Raf. (180 trees); (Site 2) sweet orange variety 'Criolla' grafted onto trifoliate orange (100 trees); and (Site 3) hybrid tangerine variety 'Nova', Citrus reticulata Hort. Ex Tan. $\times$ (Citrus paradise Macf. $\times$ Citrus tangerine Hort. Ex Tan.), grafted onto trifoliate orange (ca. 800 trees). The common weeds found around these citrus orchards were Ambrosia tenuifolia Spreng., Bidens spp. (Asteraceae), Chloris gayana Kunth, Paspalum dilatatum Poiret, Paspalum notatum Flueggé, Sorghum halepense L., Cynodon dactylon (L.), Digitaria sanguinalis L., Cenchrus echinatus L. (Poaceae), Echium plantagineum L. (Boraginaceae), Amaranthus hybridus var. quitensis (Kunth) (Amaranthaceae), Cyperus rotundus L. (Cyperaceae), Sida rhombifolia L. (Malvaceae), and Lantana camara L. (Verbenaceae). There was no weed control at sites 1 and 2 during the sampling period, whereas at site 3 , weeds were controlled monthly by mowing.

\section{Insect sampling and taxonomic identification}

Insects were collected using yellow sticky traps $(12.5 \times 10 \mathrm{~cm})$ installed at a height of $180 \mathrm{~cm}$ on the branches of citrus plants. This method enables insect activity to be measured and provides continuous sampling (Purcell, 1994). In total, 30 traps were installed, five on sweet orange 'Valencia Late', five on sweet orange 'Criolla', and 20 on hybrid tangerine 'Nova' (one trap per 30 plants, approximately). The traps were replaced monthly over a 3-year period, from October 2009 to October 2012 (36 samples in each orchard). Specimens collected from sticky traps were removed using benzene to dissolve the glue, and preserved in labeled microfuge tubes with $96 \%$ ethanol. In addition, three $50-\mathrm{m}$ long transects were established in each agroecosystem to sample insects from weeds that could serve as host plants for potential vectors of $X$. fastidiosa. On each transect, insects were collected with entomological sweep nets $(30 \mathrm{~cm}$ diameter), with 100 sweeps per transect (300 sweeps per orchard), carried out $15 \times$ during the sampling period. The collected insects were preserved in labeled microfuge tubes with $96 \%$ ethanol. Due to the different sampling efforts with yellow sticky traps and the entomological nets, the data were analyzed separately, and only the data obtained by sticky traps were used to study seasonal fluctuation.

Species were identified based on the following literature: Lawson (1931), Christensen (1942), Young (1952, 1968, 1977), Linnavuori (1959), Nielson (1968), De Long \& Freytag (1976), Remes Lenicov (1982), Barreira \& Sakakibara (2001), and Paradell \& Remes Lenicov (2005). The genitalia were prepared with a saturated $\mathrm{KOH}$ solution for observation and specific identification when necessary, and preserved in microvials with glycerin. The taxonomic 
terminology proposed by Dietrich (2005) was used. A selection of specimens was deposited in the Entomological Collection of Museo de La Plata, Argentina (MLP).

\section{Data analysis and seasonal fluctuation}

The abundance, frequency, and constancy indices were calculated for each species (Silveira Neto et al., 1976). The Shapiro-Wilk test was performed to evaluate whether a sample of the population was normally distributed (Zar, 1984). Abundance and frequency values were tested for each species. If the distribution deviated significantly from normality, 95 and 99\% confidence intervals (CI) were calculated using non-parametric methods with 1000 replicates using InfoStat v.2012 software (Di Rienzo et al., 2012). For mean abundance, the CI was calculated at 1 and $5 \%$ probability, and the species were arranged into the following classes: rare $(r)$, number of collected specimens below the lower CI limit at 1\% probability; dispersed (d), number of specimens between the lower CI limits at 1 and $5 \%$ probability; common (c), number of collected specimens within the $95 \% \mathrm{CI}$; abundant (a), number of specimens between the upper CI limits at 5 and $1 \%$ probability; and very abundant (va), number of collected specimens above the upper CI limit at 1\% probability. For mean frequency, the following classes were established by calculating the 95\% CI: infrequent (if), when the percentage of collected specimens was below the lower CI limit; frequent (f), percentage within the CI; and very frequent (vf), when the percentage of collected specimens was above the upper CI limit. The constancy of each species was calculated using the formula $\mathrm{C}=$ (number of samples where the species ' $i$ ' occurred/total number of samples $) \times 100$. Based on values obtained, the species were classified as: constant (w), when $\mathrm{C}$ was higher than 50\%; accessory (y), when C was 25-50\%; and accidental ( $\mathrm{z}$ ), when $\mathrm{C}$ was less than $25 \%$. The species with the highest faunistic indices of constancy, frequency, and abundance were called predominant species (Silveira Neto et al., 1995).

The monthly activity of predominant species collected with sticky traps was obtained. Between 2009 and 2012, the phenological stages of citrus plants were recorded monthly as follows: PS1, early sprouts flush; PS2, elongating shoots and small leaves; PS3, elongated shoots and expanding leaves; PS4, leaves attaining final size; and PS5, no new shoots, twigs, and leaves predominantly mature. This information was used to study seasonal fluctuation and evaluate the possible influence of the phenology in the orchard on insect abundance. Maximum and minimum temperature $\left({ }^{\circ} \mathrm{C}\right)$, relative humidity $(\%)$, and rainfall $(\mathrm{mm}$ cumulative) were obtained from the weather station located at the INTA Agricultural Experimental Station in Concordia, Entre Rios (less than ca. $700 \mathrm{~m}$ from the study sites). We used the Pearson product-moment correlation coefficient which estimates the association between two variables, to determine whether the magnitude of a variable changes together with the change of the second variable (Zar, 1984; Sokal \& Rohlf, 1999). The average of each climatic variable was calculated for each sampling period. These averages were correlated with the abundance values of the predominant species to determine possible association between climatic conditions and seasonal fluctuation of insects.

\section{Detection of Xylella fastidiosa}

Molecular methods were used to detect the bacterium $X$. fastidiosa because they have proved to be more sensitive and have better specificity and discriminatory capabilities than DIBA techniques (Minsavage et al., 1993, 1994).

DNA extraction from insects. Whole insects were homogenized individually in a mini bead beater (one or two 1 -min pulses) in Bashing Beads lysis tubes and $500 \mu \mathrm{l}$ CTAB buffer (2\% CTAB, 0.2\% $\beta$-mercaptoethanol, $1 \mathrm{M}$ $\mathrm{NaCl}, 20$ mM EDTA, $100 \mathrm{nM}$ Tris-HCl, pH 8). DNA was extracted from the samples using a phenol:chloroform: isoamyl alcohol (25:24:1) solution, precipitated by adding 0.1 volumes of $100 \%$ ethanol-sodium acetate $(3 \mathrm{M}, \mathrm{pH}$ 5.2), and washed and resuspended in 20-30 $\mu$ l of Milli-Q water. DNA samples were stored at $-20{ }^{\circ} \mathrm{C}$ until PCR was conducted.

DNA extraction from plants. A 3-cm piece of leaf petiole and adjacent central vein was pulverized in liquid nitrogen and the tissue powder obtained was placed in $1.5 \mathrm{ml}$ DNAzolEs (Molecular Research Center, Cincinnati, $\mathrm{OH}$, USA). Samples were mixed by inversion for $10 \mathrm{~min}$ until the tissue powder was thawed and suspended. Thereafter, the method was performed following the manufacturer's instructions, and DNA was extracted by using 1 volume of chloroform and precipitated using 1 volume of isopropanol, washed, resuspended in TE $(10 \mathrm{mM}$ Tris$\mathrm{HCl}, 1 \mathrm{mM}$ EDTA, $\mathrm{pH} 8$ ), and stored at $-20{ }^{\circ} \mathrm{C}$ until the PCRs were conducted.

Quality of DNA obtained was controlled by electrophoresis in $1 \%$ agarose (Invitrogen, Carlsbad, CA, USA) gel using $1 \times$ TAE buffer ( $40 \mathrm{mM}$ Tris-acetate, $1 \mathrm{mM}$ EDTA) and ethidium bromide $\left(0.5 \mathrm{mg} \mathrm{ml}^{-1}\right)$, and quantified using a Nanodrop CNd1000 spectrophotometer. PCRs were conducted using a primer pair especially designed for this study that amplified a 144-bp region of the gyrase $B$ gene of $X$. fastidiosa (Xyl unq F: $5^{\prime}$ GAAGGTGGTATTCGTAGC-3'; Xyl unq R: $5^{\prime}$ CATTGTTTCTTGGTAGGC- $3^{\prime}$ ). The primers were designed based on the alignment of sequences of gyr $B$ gene 
of different strains of X. fastidiosa available in GenBank (accession numbers: AE003849.1, AE009442.1, CP000941.1, CP001011.1). PCRs were carried out using a reaction mixture consisting of $0.2 \mathrm{mM}$ of each dNTP, $2 \mathrm{mM} \mathrm{MgCl}_{2}, 0.5 \mu \mathrm{M}$ of each primer, $1 \mathrm{U}$ TaqDNA polymerase (Invitrogen), in a PCR buffer $1 \times(20 \mathrm{mM}$ Tris$\mathrm{HCl}, \mathrm{pH}$ 8.4, $50 \mathrm{nM} \mathrm{KCl}$ ), and $200 \mathrm{ng}$ sample DNA. The total volume per reaction was $30 \mu \mathrm{l}$. A Mastercycler Gradient thermocycler (Eppendorf, Hamburg, Germany) was used with the following program: denaturation for $2 \mathrm{~min}$ at $94{ }^{\circ} \mathrm{C}$, followed by 35 cycles of $20 \mathrm{~s}$ at $94{ }^{\circ} \mathrm{C}, 30 \mathrm{~s}$ at $57^{\circ} \mathrm{C}$, and $1 \mathrm{~min}$ at $72{ }^{\circ} \mathrm{C}$, with a final extension at $72{ }^{\circ} \mathrm{C}$ for $5 \mathrm{~min}$. A reaction mixture without template DNA was used as negative control and a DNA from CVC-affected citrus was used as positive control. PCR products were visualized by electrophoresis in $1.8 \%$ agarose gel with ethidium bromide $\left(0.5 \mathrm{mg} \mathrm{ml}^{-1}\right)$ using a 50 -bp ladder (Productos Bio-Lógicos, Bernal, Argentina) as size standard marker. A sample was considered positive for the presence of $X$. fastidiosa if a fragment of ca. 150 bp was visualized under UV light. Fifteen random samples were corroborated by sequencing.

Additionally, real-time PCR was performed using a SmartCycler (Cepheid, Sunnyvale, CA, USA) with a final reaction volume of $25 \mu \mathrm{l}$, using $12.5 \mu \mathrm{l}$ PerfeCta SYBRGreen FastMix 2x concentrated (Quanta Biosciences, Beverly, MA, USA), $1.25 \mu \mathrm{l}$ of each primer $(10 \mu \mathrm{M}), 8 \mu \mathrm{l}$ Mili-Q water, and $600 \mathrm{ng}$ sample DNA. The cycling consisted of $30 \mathrm{~s}$ at $95{ }^{\circ} \mathrm{C}, 30$ cycles of $95{ }^{\circ} \mathrm{C}$ for $20 \mathrm{~s}, 56^{\circ} \mathrm{C}$ for $25 \mathrm{~s}$, and $72{ }^{\circ} \mathrm{C}$ for $20 \mathrm{~s}$. At the end of the reaction, the temperature was increased from 72 to $99{ }^{\circ} \mathrm{C}$ at a rate of $0.1{ }^{\circ} \mathrm{C} \mathrm{s}^{-1}$, and fluorescence was measured every $1{ }^{\circ} \mathrm{C}$ increase for construction of the melting curve. Samples with cycle threshold $(\mathrm{Ct})$ less than 29 were considered positive. In all real-time PCR reactions, a mixture without template DNA was used as negative control and a plasmid carrying the fragment of the gyrase $B$ gene of $X$. fastidiosa was used as positive control.

\section{Transmission assay}

Insects were collected from the 'Valencia Late' sweet orange orchard (Site 1) and transferred in groups of five adults into individual plastic cages containing a CVCinfected source citrus plant for a 48- to 72-h acquisition access period (AAP). The source plant was confirmed positive for X. fastidiosa by PCR as described above. After the AAP, live insects were transferred individually to a caged healthy sweet orange plant which was confirmed negative for X. fastidiosa by PCR, for a 72- to 96-h inoculation access period (IAP). Small test plants were used because they are more susceptible to infection by $X$. fastidiosa than larger plants, and the bacterium is more easily detected
(Brlansky et al., 2002). After the IAP, the insects were preserved in absolute ethanol at $4{ }^{\circ} \mathrm{C}$ until the PCR conduct. One month later, the test plants were analyzed for $X$. fastidiosa by PCR.

\section{Results}

Leafhoppers and treehoppers in citrus agroecosystems

During the sampling period, 6052 insects belonging to six families of Auchenorrhyncha were collected. Cicadellidae and Membracidae were the most abundant families (Table 1). Forty-one species of Cicadellidae (4 631 individuals) and two species of Membracidae (162 individuals) were identified (Tables 2 and 3 ).

Twenty-seven species (4 409 individuals) were collected with yellow sticky traps on citrus plants (Table 2). Although the number of insects collected in each orchard differed, due to the different sampling efforts, species richness was similar in all three orchards. Five species had the highest faunistic indices: Scaphytopius bolivianus Oman was the most abundant ( 1828 collected individuals) and predominant in the three citrus orchards studied, followed by Frequenamia spiniventris (Linnavuori) (1 265 collected individuals). The leafhopper Molomea lineiceps Young was collected from all citrus varieties studied, although it was only predominant in the Nova tangerine orchard. Tapajosa rubromarginata (Signoret) was collected from all three citrus agroecosystems studied, and was a predominant species in orchards of both sweet orange varieties. Bucephalogonia xanthophis (Berg) was collected from all citrus varieties, but was only predominant in the Valencia Late sweet orange orchard (Table 2).

Thirty-two species (384 specimens) were collected with entomological nets from weeds surrounding citrus (Table 3). Although at site 3 weeds were scarce and controlled monthly and the number of collected insects was lower, species richness was similar at all three sites. The treehopper Cyphonia clavigera (Fabricius) and the leafhopper Hortensia similis (Walker) were predominant on weeds

Table 1 Number of insects per family of Auchenorrhyncha collected from Citrus Variegated Chlorosis-affected citrus agroecosystems over a 3-year period

\begin{tabular}{lc}
\hline Family & No. specimens \\
\hline Cicadidae & 1 \\
Cercopidae & 10 \\
Cicadellidae & 5505 \\
Delphacidae & 49 \\
Flatidae & 29 \\
Membracidae & 458 \\
Total & 6052 \\
\hline
\end{tabular}


Table 2 Auchenorrhyncha collected by yellow sticky traps on Citrus Variegated Chlorosis-affected citrus plants over a 3-year period. Site 1, sweet orange 'Valencia Late'; Site 2, sweet orange 'Criolla'; Site 3, hybrid tangerine 'Nova'. A, abundance (r, rare; d, dispersed; c, common; va, very abundant); F, frequency (if, infrequent; $f$, frequent; vf, very frequent); C, constancy ( $w$, constant; $y$, accessory; $\mathrm{z}$, accidental)

\begin{tabular}{|c|c|c|c|c|c|c|c|c|c|c|c|c|c|c|}
\hline \multirow{2}{*}{\multicolumn{2}{|c|}{ Family/Subfamily/Species }} & & \multicolumn{4}{|c|}{ Site 1} & \multicolumn{4}{|c|}{ Site 2} & \multicolumn{4}{|l|}{ Site 3} \\
\hline & & & \multirow{2}{*}{$\frac{\mathrm{n}}{74}$} & \multirow{2}{*}{$\begin{array}{l}\text { A } \\
\text { va }\end{array}$} & \multirow{2}{*}{$\begin{array}{l}\mathrm{F} \\
\mathrm{vf}\end{array}$} & \multirow{2}{*}{$\frac{\mathrm{C}}{\mathrm{w}}$} & \multirow{2}{*}{$\frac{\mathrm{n}}{15}$} & \multirow{2}{*}{ A } & \multirow{2}{*}{$\frac{\mathrm{F}}{\mathrm{f}}$} & \multirow{2}{*}{$\frac{\mathrm{C}}{\mathrm{z}}$} & \multirow{2}{*}{ n } & \multirow{2}{*}{$\frac{A}{d}$} & \multirow{2}{*}{$\frac{F}{f}$} & \multirow{2}{*}{$\frac{\mathrm{C}}{\mathrm{z}}$} \\
\hline CICADELLIDAE & Cicadellinae & $\begin{array}{l}\text { Bucephalogonia xanthophis } \\
\text { (Berg) }\end{array}$ & & & & & & & & & & & & \\
\hline & & $\begin{array}{l}\text { Diedrocephala bimaculata } \\
\text { (Gmelin) }\end{array}$ & 13 & c & $\mathrm{f}$ & $\mathrm{z}$ & 16 & c & $\mathrm{f}$ & z & 7 & c & $\mathrm{f}$ & $\mathrm{z}$ \\
\hline & & Hortensia similis (Walker) & 1 & $\mathrm{r}$ & if & $\mathrm{z}$ & & & & & & & & \\
\hline & & Macugonalia cavifrons (Stål) & & & & & & & & & 1 & $\mathrm{r}$ & if & z \\
\hline & & Macugonalia sobrina (Stål) & 2 & $\mathrm{r}$ & if & $\mathrm{z}$ & 6 & $\mathrm{r}$ & if & $\mathrm{z}$ & 1 & $\mathrm{r}$ & if & z \\
\hline & & Molomea lineiceps Young & 30 & c & $\mathrm{f}$ & $\mathrm{y}$ & 39 & va & vf & $\mathrm{y}$ & 633 & va & vf & $\mathrm{w}$ \\
\hline & & Oragua triplehorni Young & 3 & $\mathrm{r}$ & if & $\mathrm{z}$ & 2 & $\mathrm{r}$ & if & $\mathrm{z}$ & 1 & $\mathrm{r}$ & if & $\mathrm{z}$ \\
\hline & & Pawiloma victima (Germar) & 1 & $\mathrm{r}$ & if & $\mathrm{z}$ & & & & & & & & \\
\hline & & Plesiommata mollicella (Fowler) & & & & & 1 & $\mathrm{r}$ & if & $\mathrm{z}$ & 4 & d & $\mathrm{f}$ & $\mathrm{z}$ \\
\hline & & Sonesimia grossa (Signoret) & & & & & & & & & 1 & $\mathrm{r}$ & if & $\mathrm{z}$ \\
\hline & & $\begin{array}{l}\text { Tapajosa rubromarginata } \\
\text { (Signoret) }\end{array}$ & 60 & va & vf & $\mathrm{w}$ & 101 & va & vf & $\mathrm{w}$ & 82 & c & $\mathrm{f}$ & $\mathrm{w}$ \\
\hline & Coelidiinae & Coelidia spec. & 1 & $\mathrm{r}$ & if & $\mathrm{z}$ & & & & & & & & \\
\hline & Deltocephalinae & Balclutha spec. 1 & 14 & c & f & $\mathrm{z}$ & & & & & & & & \\
\hline & & $\begin{array}{l}\text { Dalbulus maidis (De Long \& } \\
\text { Wolcott) }\end{array}$ & 1 & $\mathrm{r}$ & if & $\mathrm{z}$ & 2 & $\mathrm{r}$ & if & z & 2 & $\mathrm{r}$ & if & $\mathrm{z}$ \\
\hline & & $\begin{array}{l}\text { Frequenamia spiniventris } \\
\text { (Linnavuori) }\end{array}$ & 139 & va & vf & $\mathrm{w}$ & 140 & va & $\mathrm{vf}$ & $\mathrm{w}$ & 986 & va & vf & $\mathrm{w}$ \\
\hline & & Scaphytopius bolivianus Oman & 156 & va & vf & $\mathrm{w}$ & 71 & va & $\mathrm{vf}$ & $\mathrm{w}$ & 1601 & va & vf & $\mathrm{w}$ \\
\hline & & Spangbergiella vulnerata (Uhler) & 1 & $\mathrm{r}$ & if & $\mathrm{z}$ & & & & & & & & \\
\hline & & Stirellus spec. & & & & & & & & & 1 & $\mathrm{r}$ & if & $\mathrm{z}$ \\
\hline & Iassinae & $\begin{array}{l}\text { Curtara concava De Long \& } \\
\text { Freytag }\end{array}$ & & & & & 2 & $\mathrm{r}$ & if & $\mathrm{z}$ & & & & \\
\hline & & $\begin{array}{l}\text { Curtara pagina De Long \& } \\
\text { Freytag }\end{array}$ & & & & & & & & & 1 & $\mathrm{r}$ & if & $\mathrm{z}$ \\
\hline & & $\begin{array}{l}\text { Curtara samera De Long \& } \\
\text { Freytag }\end{array}$ & 6 & $\mathrm{~d}$ & if & $\mathrm{z}$ & 19 & c & $\mathrm{f}$ & z & 1 & $\mathrm{r}$ & if & $\mathrm{z}$ \\
\hline & Megophthalminae & Agalliana ensigera Oman & 19 & c & $\mathrm{f}$ & $\mathrm{z}$ & & & & & 2 & $\mathrm{r}$ & if & $\mathrm{z}$ \\
\hline & & Agalliana sticticollis (Stål) & & & & & 2 & $\mathrm{r}$ & if & $\mathrm{z}$ & 4 & d & $\mathrm{f}$ & $\mathrm{z}$ \\
\hline & Ledrinae & Xerophloea viridis (Fabricius) & 8 & $\mathrm{~d}$ & if & $\mathrm{z}$ & 1 & $\mathrm{r}$ & if & $\mathrm{z}$ & 3 & $\mathrm{~d}$ & $\mathrm{f}$ & $\mathrm{z}$ \\
\hline & Typhlocybinae & Protalebrella brasiliensis (Baker) & 6 & $\mathrm{~d}$ & if & $\mathrm{z}$ & 4 & $\mathrm{r}$ & if & $\mathrm{z}$ & 2 & $\mathrm{r}$ & if & $\mathrm{z}$ \\
\hline \multirow{2}{*}{\multicolumn{2}{|c|}{ MEMBRACIDAE }} & Cyphonia clavigera (Fabricius) & 10 & c & $\mathrm{f}$ & $\mathrm{z}$ & 62 & va & vf & $\mathrm{y}$ & 12 & c & $\mathrm{f}$ & $\mathrm{z}$ \\
\hline & & Entylia carinata (Forster) & 18 & c & $\mathrm{f}$ & $\mathrm{z}$ & 12 & $c$ & $\mathrm{f}$ & $\mathrm{z}$ & 3 & c & $\mathrm{f}$ & $\mathrm{z}$ \\
\hline \multicolumn{3}{|c|}{ Total number of collected specimens } & 563 & & & & 495 & & & & 3351 & & & \\
\hline \multicolumn{3}{|c|}{ Species richness } & 20 & & & & 17 & & & & 21 & & & \\
\hline
\end{tabular}

of Valencia Late sweet orange orchard, whereas T. rubromarginata was the predominant species on weeds of both sweet orange orchards. No species was predominant in weeds surrounding the Nova tangerine orchard (Table 3).

\section{Seasonal fluctuation of predominant species}

The abundance of $S$. bolivianus was positively correlated with the maximum and minimum temperatures. A similar but less pronounced trend was observed for $F$. spiniventris (Table 4). During the sampling period, the populations of both species were higher in summer and lower in winter (Figure 1A). The abundance of M. lineiceps, T. rubromarginata, and B. xanthophis was not correlated with the climatic variables analyzed (Table 4). Two population increases per year (Figure 1B) were observed for these species: one in summer (December and January), and another in winter (July, August, and September). The phenological data from the three orchards showed that these citrus varieties had two sprouting periods over the year, a smaller one in summer and a larger one in winter, coinciding with 
Table 3 Auchenorrhyncha collected by entomological sweep net on weeds surrounding citrus orchards over a 3-year period. Site 1, sweet orange 'Valencia Late'; Site 2, sweet orange 'Criolla'; Site 3, hybrid tangerine 'Nova'. A, abundance (r, rare; d, dispersed; c, common; va, very abundant); F, frequency (if, infrequent; f, frequent; vf, very frequent); C, constancy (w, constant; $y$, accessory; $z$, accidental)

\begin{tabular}{|c|c|c|c|c|c|c|c|c|c|c|c|c|c|c|}
\hline \multirow{2}{*}{\multicolumn{2}{|c|}{ Family/subfamily/species }} & & \multicolumn{4}{|c|}{ Site 1} & \multicolumn{4}{|c|}{ Site 2} & \multicolumn{4}{|c|}{ Site 3} \\
\hline & & & $\mathrm{n}$ & A & $\mathrm{F}$ & $\mathrm{C}$ & $\mathrm{n}$ & A & $\mathrm{F}$ & $\mathrm{C}$ & $\mathrm{n}$ & A & $\mathrm{F}$ & $\mathrm{C}$ \\
\hline \multirow[t]{31}{*}{ CICADELLIDAE } & Cicadellinae & Balacha melanocephala (Signoret) & & & & & 1 & $\mathrm{r}$ & if & $\mathrm{z}$ & & & & \\
\hline & & Bucephalogonia xanthophis (Berg) & 3 & c & $\mathrm{f}$ & z & & & & & & & & \\
\hline & & Dechacona missionum (Berg) & & & & & 1 & $\mathrm{r}$ & if & $\mathrm{z}$ & & & & \\
\hline & & Diedrocephala bimaculata (Gmelin) & 8 & c & $\mathrm{f}$ & $\mathrm{y}$ & 1 & $\mathrm{r}$ & if & $\mathrm{z}$ & 3 & c & $\mathrm{f}$ & $\mathrm{z}$ \\
\hline & & Hortensia similis (Walker) & 27 & va & vf & $\mathrm{w}$ & 6 & c & $\mathrm{f}$ & $\mathrm{y}$ & & & & \\
\hline & & Molomea lineiceps Young & & & & & & & & & 1 & $\mathrm{r}$ & if & $\mathrm{z}$ \\
\hline & & Oragua triplehorni Young & & & & & 3 & c & $\mathrm{f}$ & $\mathrm{z}$ & & & & \\
\hline & & Plesiommata mollicella (Fowler) & 17 & va & $\mathrm{vf}$ & $\mathrm{y}$ & 7 & c & $\mathrm{f}$ & z & 3 & c & $\mathrm{f}$ & $\mathrm{z}$ \\
\hline & & Sibovia sagata (Signoret) & 2 & $\mathrm{~d}$ & if & $\mathrm{z}$ & & & & & & & & \\
\hline & & Sonesimia grossa (Signoret) & 2 & $\mathrm{~d}$ & if & $\mathrm{z}$ & 4 & c & $\mathrm{f}$ & $\mathrm{y}$ & & & & \\
\hline & & Syncharina argentina (Berg) & & & & & 1 & $\mathrm{r}$ & if & $\mathrm{z}$ & 1 & $\mathrm{r}$ & if & $\mathrm{z}$ \\
\hline & & Tapajosa rubromarginata (Signoret) & 22 & va & vf & $\mathrm{w}$ & 11 & va & vf & $\mathrm{w}$ & 12 & va & vf & $\mathrm{y}$ \\
\hline & Deltocephalinae & Amplicephalus marginellanus (Metcalf) & 2 & $\mathrm{~d}$ & if & $\mathrm{z}$ & & & & & 1 & $\mathrm{r}$ & if & $\mathrm{z}$ \\
\hline & & Amplicephalus spec. & 1 & $\mathrm{r}$ & if & $\mathrm{z}$ & 3 & c & $\mathrm{f}$ & $\mathrm{z}$ & 3 & c & $\mathrm{f}$ & $\mathrm{z}$ \\
\hline & & Atanus spec. & & & & & & & & & 1 & $\mathrm{r}$ & if & $\mathrm{z}$ \\
\hline & & Balclutha spec. 1 & & & & & 55 & va & vf & $\mathrm{z}$ & 1 & $\mathrm{r}$ & if & z \\
\hline & & Balclutha spec. 2 & 27 & va & vf & z & & & & & & & & \\
\hline & & Clorindaia brasileira Zahniser & 1 & $\mathrm{r}$ & if & $\mathrm{z}$ & & & & & 2 & c & $\mathrm{f}$ & z \\
\hline & & Clorindaia hecaloides Linnavuori & & & & & 1 & $\mathrm{r}$ & if & $\mathrm{z}$ & & & & \\
\hline & & Exitianus obscurinervis (Stål) & 1 & $\mathrm{r}$ & if & $\mathrm{z}$ & & & & & 5 & va & vf & $\mathrm{z}$ \\
\hline & & Faltala brachyptera Oman & 1 & $\mathrm{r}$ & if & z & 2 & c & $\mathrm{f}$ & $\mathrm{z}$ & & & & \\
\hline & & Haldorus sexpunctatus (Berg) & 12 & va & vf & $\mathrm{z}$ & 6 & c & $\mathrm{f}$ & $\mathrm{z}$ & & & & \\
\hline & & Mendozellus asunctia Cheng & 2 & $\mathrm{~d}$ & if & $\mathrm{z}$ & & & & & & & & \\
\hline & & Mendozellus dubius (Linnavuori) & & & & & & & & & 1 & $\mathrm{r}$ & if & $\mathrm{z}$ \\
\hline & & Scaphytopius bolivianus Oman & & & & & & & & & 8 & va & vf & $\mathrm{y}$ \\
\hline & & Spangbergiella vulnerata (Uhler) & 3 & c & $\mathrm{f}$ & z & 2 & c & $\mathrm{f}$ & $\mathrm{z}$ & 1 & $\mathrm{r}$ & if & $\mathrm{z}$ \\
\hline & Megophthalminae & Agalliana ensigera Oman & 1 & $\mathrm{r}$ & if & z & 2 & c & $\mathrm{f}$ & $\mathrm{z}$ & 4 & va & vf & $\mathrm{z}$ \\
\hline & & Agalliana sticticollis (Stål) & 1 & $\mathrm{r}$ & if & $\mathrm{z}$ & 16 & va & vf & $\mathrm{y}$ & 11 & va & vf & $\mathrm{y}$ \\
\hline & Ledrinae & Xerophloea viridis (Fabricius) & 1 & $\mathrm{r}$ & if & $\mathrm{z}$ & 4 & $c$ & $\mathrm{f}$ & $\mathrm{y}$ & 3 & $c$ & $\mathrm{f}$ & $\mathrm{z}$ \\
\hline & Typhlocybinae & Empoasca spec. & & & & & & & & & 1 & $\mathrm{r}$ & if & z \\
\hline & & Protalebrella brasiliensis (Baker) & 8 & $\mathrm{c}$ & $\mathrm{f}$ & $\mathrm{y}$ & 4 & $c$ & $\mathrm{f}$ & $\mathrm{z}$ & 5 & va & vf & $\mathrm{y}$ \\
\hline \multicolumn{2}{|c|}{ MEMBRACIDAE } & Cyphonia clavigera (Fabricius) & 30 & va & vf & $\mathrm{w}$ & 14 & va & vf & $\mathrm{y}$ & 1 & $\mathrm{r}$ & if & $\mathrm{z}$ \\
\hline \multicolumn{3}{|c|}{ Total number of collected specimens } & 172 & & & & 144 & & & & 68 & & & \\
\hline \multicolumn{3}{|c|}{ Species richness } & 21 & & & & 20 & & & & 20 & & & \\
\hline
\end{tabular}

the periods of population increase of M. lineiceps, T. rubromarginata, and B. xanthophis.

\section{Detection of Xylella fastidiosa}

Of the 150 insects tested, $33.3 \%$ were positive for $X$. fastidiosa by conventional PCR and real-time PCR. Eleven species of leafhoppers and treehoppers were positive for $X$. fastidiosa (Table 5). All predominant species both on citrus plants and on weeds surrounding the citrus plants were positive. Plesiommata mollicella (Fowler), F. spiniventris, H. similis, S. bolivianus, and T. rubromarginata were positive by both detection methods, whereas B. xanthophis, Dechacona missionum (Berg), M. lineiceps, C. clavigera, and Entylia carinata (Forster) were positive by conventional PCR, and Curtara samera De Long \& Freytag was only positive by real-time PCR. The conventional PCR method was more effective, being cheaper and easier to standardize than the real-time PCR method.

\section{Transmission of Xylella fastidiosa}

Only a few insects survived the AAP on infected source plants. A single specimen of C. clavigera and D. missionum, and five specimens of T. rubromarginata were 
Table 4 Pearson correlation coefficients between climatic parameters and abundance of five predominant species

\begin{tabular}{lcccc}
\hline Predominant species & Maximum temperature & Minimum temperature & Relative humidity & Rainfall \\
\hline Bucephalogonia xanthophis & -0.48 & -0.44 & 0.23 & 0.03 \\
Frequenamia spiniventris & 0.57 & 0.61 & 0.02 & 0.3 \\
Molomea lineiceps & -0.12 & -0.13 & -0.01 & 0.1 \\
Scaphytopius bolivianus & 0.75 & 0.79 & -0.22 & 0.32 \\
Tapajosa rubromarginata & 0.05 & 0.13 & 0.07 & 0.22 \\
\hline
\end{tabular}
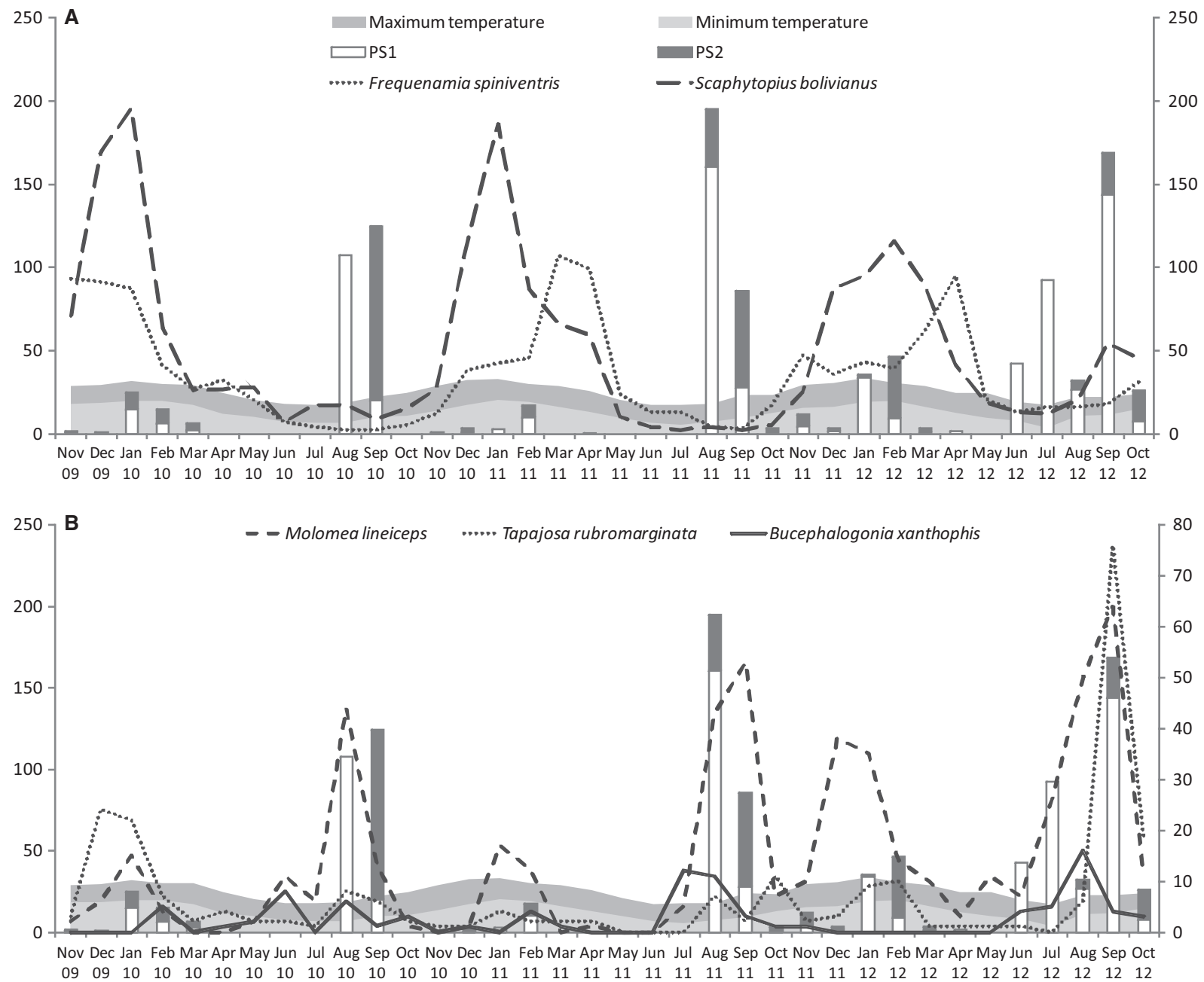

Figure 1 Seasonal fluctuation (from November 2009 to October 2012) of five species of leafhopper in Citrus Variegated Chlorosis-affected citrus orchards. Y-axes left: minimum and maximum temperatures $\left({ }^{\circ} \mathrm{C}\right)$ and abundance of phenological stages (PS) of citrus [number of citrus plants in PS1 (early sprouts flush) and PS2 (elongating shoots and small leaves)]; Y-axes right: abundance of each species of leafhopper (number of collected leafhoppers monthly) (A) Scaphytopius bolivianus and Frequenamia spiniventris and (B) Bucephalogonia xanthophis, Molomea lineiceps, and Tapajosa rubromarginata.

used in the experimental transmission assay. These three analyzed species tested positive for $X$. fastidiosa after the AAP. The seven test plants tested positive for $X$. fastidiosa by PCR after IAP (a month later), although no symptom was observed. Electrophoresis analysis of PCR amplification products obtained from insect and test-plant samples 
Table 5 Number of samples per species testing positive for Xylella fastidiosa by conventional PCR and real-time PCR

\begin{tabular}{|c|c|c|c|c|}
\hline \multicolumn{3}{|c|}{ Family/Subfamily/Species } & \multirow{2}{*}{$\frac{\text { No. samples }}{11}$} & \multirow{2}{*}{$\frac{\text { No. positive }}{4}$} \\
\hline CICADELLIDAE & Cicadellinae & Bucephalogonia xanthophis & & \\
\hline & & Dechacona missionum & 1 & 1 \\
\hline & & Diedrocephala bimaculata & 3 & 0 \\
\hline & & Hortensia similis & 5 & 3 \\
\hline & & Molomea lineiceps & 37 & 16 \\
\hline & & Plesiommata mollicella & 8 & 5 \\
\hline & & Tapajosa rubromarginata & 20 & 6 \\
\hline & & Sonesimia grossa & 4 & 0 \\
\hline & Deltocephalinae & Frequenamia spiniventris & 11 & 5 \\
\hline & & Scaphytopius bolivianus & 13 & 3 \\
\hline & Iassinae & Curtara samera & 2 & 1 \\
\hline & Megophthalminae & Agalliana ensigera & 5 & 0 \\
\hline & & Agalliana sticticollis & 5 & 0 \\
\hline \multirow[t]{2}{*}{ MEMBRACIDAE } & & Cyphonia clavigera & 20 & 4 \\
\hline & & Entylia carinata & 5 & 2 \\
\hline Total & & & 150 & 50 \\
\hline
\end{tabular}

showed a 150-bp fragment, which indicates that all samples tested positive for $X$. fastidiosa, suggesting that transmission of the bacterium by the three hemipteran species was successful. The two control plants were negative for $X$. fastidiosa by PCR.

\section{Discussion}

The number of citrus plants, and thus the number of sticky traps placed, as well as the diversity and abundance of weeds could influence the number of insects collected in each citrus agroecosystems. Therefore, an agroecosystem with low-intensity control and greater diversity of weeds will have higher numbers of leafhoppers (Mizell et al., 2003). Species richness was similar when comparing the citrus orchards or weeds among sites, but it was higher in the weeds than in citrus orchards. However, the shared species between citrus orchards and weeds indicate that most of the species of leafhoppers and treehoppers located in orchards are also frequent on weeds, which therefore may function as reservoir for these insects.

All predominant species collected in citrus and weeds tested positive for $X$. fastidiosa by molecular methods. However, detection of the bacterium in the body of insects does not mean that the species are vectors, because it could be only transient. Plant-to-plant transmission tests are necessary to undoubtedly demonstrate that a given insect species is a vector of $X$. fastidiosa.

Among the Cicadellinae, B. xanthophis, one of the main vectors of $X$. fastidiosa in Brazil (Marucci et al., 2008), tested positive in this study by conventional PCR, and also positive by DIBA on specimens from the Misiones Province (de Coll et al., 2000). Molomea lineiceps also tested positive for $X$. fastidiosa by conventional PCR, and has been reported as abundant on citrus plants in Argentina and Brazil (Azevedo Filho \& Carvalho, 2006; Dellapé et al., 2013; Fabril et al., 2014). Another species of the genus, Molomea cincta (Signoret), has been reported by Fundecitrus (1999) and Yamamoto et al. (2000) as a potential vector of $X$. fastidiosa in Brazil. Tapajosa rubromarginata was an abundant species on citrus plants and weeds and positive for $X$. fastidiosa after the transmission assay, so could therefore be acting as vector of the bacterium in the orchards studied. Tapajosa rubromarginata is widely distributed in Argentina (Paradell et al., 2012) and considered a pest of the main agricultural crops, causing direct damage by feeding and oviposition (Remes Lenicov et al., 1998). Hortensia similis was predominant in weeds and positive for X. fastidiosa by both molecular methods; de Coll et al. (2000) detected the bacterium by DIBA on specimens from Misiones. According to Redak et al. (2004), most Cicadellinae species have shown ability to acquire and transmit $X$. fastidiosa because of their xylem-fluid feeding habit.

The deltocephaline leafhoppers S. bolivianus and $F$. spiniventris were predominant species in all the studied citrus orchards, and had not been associated with $X$. fastidiosa until this study, which showed them to be positive by PCR and real-time PCR methods. The treehopper C. clavigera was an abundant species on weeds and positive for X. fastidiosa after the transmission assay; another 
species of Membracidae, E. carinata, was also positive by molecular methods. De Coll et al. (2000) detected the bacterium in this species by serological methods. Furthermore, there are reports of Membracidae species that can transmit other $X$. fastidiosa strains to oak trees in the USA (Zhang et al., 2011). Research on the feeding behavior demonstrated that species, which do not primarily feed on xylem fluid, may nonetheless occasionally ingest xylem sap, as a means to compensate for either desiccation or osmotic stress caused by ingestion of phloem sap (Cull \& van Emden, 1977; Powell \& Hardie, 2002). This could explain why phloem-fluid feeder species of the Deltocephalinae subfamily and treehopper species (Membracidae) were positive for $X$. fastidiosa in this study.

Among the non-predominant species, Sonesimia grossa (Signoret) is a vector of CVC in Brazil with about 1.5\% transmission efficiency (Fundecitrus, 1999; Redak et al., 2004), and was positive for $X$. fastidiosa by DIBA in Misiones Province (de Coll et al., 2000). However, S. grossa was negative for the bacterium in this study, which may be related to the low number of specimens analyzed and to the fact that they were not collected directly from citrus plants, but from weeds surrounding the citrus. Plesiommata mollicella was positive by both molecular methods in this study, but it has not been previously reported as a vector of $X$. fastidiosa, although a closely related species, Plesiommata corniculata Young, is a known vector of CVC in Brazil (Krugner et al., 2000). Sibovia sagata (Signoret) and C. samera were positive for X. fastidiosa by DIBA (de Coll et al., 2000), and the latter also by molecular methods in this study. These species, along with D. missionum, which was positive after the transmission assay, should be considered as potential vectors of CVC in the area.

Regarding seasonal fluctuation of the predominant species, S. bolivianus and F. spiniventris showed population increases during the summer, possibly due to the favorable weather conditions (de Coll et al., 1993; Yamamoto \& Lopes, 2004). According to Dellapé et al. (2013), temperature increases coincide with increases in species abundance. Moreover, B. xanthophis, M. lineiceps, and T. rubromarginata populations increased twice a year, once in summer and once in winter, which could be due to the increase in early shoots of the citrus plants, which in turn increases the attractiveness for leafhopper species, as suggested by Purcell (1975) and Marucci et al. (2004). The preference of B. xanthophis, M. lineiceps, and T. rubromarginata for young growing shoots of citrus plants could be relevant in the processes of acquisition and inoculation of the bacterium because younger leaves are more susceptible to infection by $X$. fastidiosa than older leaves (Purcell, 1981; Cornara \& Porcelli, 2014). In citrus orchards, the population density of vector insects is another important factor. Insects can have relatively low transmission efficiency, but during favorable periods, when populations of insects are larger, the probability of infection increases (Purcell, 1985; Lopes, 1999; Krugner et al., 2014; EFSA, 2015; Stancanelli et al., 2015).

According to Hopkins \& Purcell (2002) X. fastidiosa could become a serious threat in areas where a vector exists. Several species tested positive for $X$. fastidiosa in this study, and showed population increases related to the weather or citrus plant conditions. Those species with the highest population densities during the sprouting period must be considered the most relevant vectors of CVC in the citrus-growing areas from Argentina.

\section{Acknowledgements}

We thank P. Dellapé (Museo de La Plata, La Plata, Argentina) for critical reading of the manuscript, and N. Costa, J.P.R. Bouvet, J. Telayna, R. Garin (INTA Agricultural Experimental Station, Concordia, Entre Rios), J.M. Marengo, L. Argüello, and D. Valdez La Hens (Laboratory of Molecular Microbiology, UNQ) for assistance with the laboratory work and field surveys. This study was supported by the Consejo Nacional de Investigaciones Científicas y Técnicas (CONICET), Grant N630 Universidad Nacional de La Plata, Argentina, and SECYT/ ANPCYT-PICT-CABBIO Grant 00125, Universidad Nacional de Quilmes, Argentina.

\section{References}

Almeida RPP \& Purcell AH (2003) Transmission of Xylella fastidiosa to grapevines by Homalodisca coagulata (Hemiptera, Cicadellidae). Journal of Economic Entomology 96: 265-271.

Almeida RPP \& Purcell AH (2006) Patterns of Xylella fastidiosa colonization on the precibarium of sharpshooter vectors relative to transmission to plants. Annals of the Entomological Society of America 99: 884-890.

Álvarez DG, Pérez AH, Díaz JS, Maestre MH \& Beltrán JH (2011) Transmisión del virus del mosaico suave del ñame a Dioscorea rotundata (Dioscoreaceae) por Oncometopia sp. (Cicadellidae). Revista Colombiana de Entomología 37: 77-79.

Azevedo Filho WS \& Carvalho GS (2006) Cigarrinhas de Citros no Rio Grande do Sul: Taxonomia. Edipucrs, Porto Alegre, Brazil.

Barreira RL \& Sakakibara AM (2001) Duas species novas de Cyphonia Laporte da regiao central do Brasil (Homoptera, Membracidae, Smiliinae). Revista Brasileira de Zoologia 18: 239-244.

Beltrán VM, Cáceres S, Zubrzycki H, Ploper D, Willink E \& Jaldo H (2004) CVC Associated Vectors in Valencia Orange of Corrientes, Argentina. Proceedings of the 10th International Citrus Congress, Agadir, Morocco, pp. 75-83. 
Brlansky RH, Damsteegt VD \& Hartung JS (2002) Transmission of the citrus variegated chlorosis bacterium Xylella fastidiosa with the sharpshooter Oncometopia nigricans. Plant Disease 86: 1237-1239.

Chen J, Chang CJ, Jarret RL \& Gawel N (1992) Genetic variation among Xylella fastidiosa strains. Phytopathology 82: 973-977.

Christensen JR (1942) Nota sobre Agalliana ensigera Oman (Homoptera). Notas del Museo de La Plata VII 55: 27-38.

de Coll OR, Agostini JP, Timmer LW \& Brlansky RH (1993) Survey of sharpshooters in citrus orchards with decline diseases in misiones, argentina. Proceedings of the 12th International Organization of Citrus Virologists Conference, pp. 320-326.

de Coll OR, Remes LA, Agostini J \& Paradell S (2000) Detection of Xylella fastidiosa in weeds and sharpshooters in orange groves affected with Citrus Variegated Chlorosis in Misiones, Argentina. Proceedings of the 14th International Organization of Citrus Virologists Conference, pp. 216-222.

Cornara D \& Porcelli F (2014) Observations on the biology and ethology of Aphrophoridae: Philaenus spumarius in the Salento Peninsula. Proceedings of the International Symposium on the European Outbreak of Xylella fastidiosa in Olive, Gallipoli, Locorotondo, Italy, p. 32.

Costa N, Plata MI, Garrán SM \& Mika R (2009) Detección de clorosis variegada de los cítricos (CVC) en el departamento de Concordia, provincia de Entre Ríos. XIII Jornadas Fitosanitarias Argentinas, Santiago del Estero: E021.

da Costa PI, Franco CF, Miranda VS, Teixeira DC \& Hartung JS (2000) Strains of Xylella fastidiosa rapidly distinguished by arbitrarily primed-PCR. Current Microbiology 40: 279-282.

Cull DC \& van Emden HF (1977) The effect on Aphis fabae of diel changes in their food quality. Physiological Entomology 2: 109-115.

De Long DM \& Freytag PH (1976) Studies of the world Gyponinae (Homoptera-Cicadellidae). A synopsis of the genus Curtara. Brenesia 7: 1-97.

Dellapé G, Bouvet JP \& Paradell SL (2013) Diversity of Cicadomorpha (Hemiptera: Auchenorrhyncha) in citrus orchards in Northeastern Argentina. Florida Entomologist 96: 11251134.

Di Rienzo JA, Casanoves F, Balzarini MG, Gonzalez L, Tablada M \& Robledo CW (2012) InfoStat v.2012. Grupo InfoStat, FCA, Universidad Nacional de Córdoba, Córdoba City, Argentina. Available at: http://www.infostat.com.ar (accessed 19 July 2015).

Dietrich CH (2005) Keys to the families of Cicadomorpha and subfamilies and tribes of Cicadellidae (Hemiptera: Auchenorrhyncha). Florida Entomologist 88: 502-517.

EFSA (European Food Safety Authority) (2015) Scientific opinion on the risks to plant health posed by Xylella fastidiosa in the EU territory, with the identification and evaluation of risk reduction options. EFSA Journal 13: 1-262.

EPPO (European and Mediterranean Plant Protection Organization) (2015) Annual Report 2014 and Council Recommendations. EPPO Bulletin 45: 479-492.

Fabril PE, Azevedo Filho WS \& Pauletti GF (2014) Análise faunística e flutuação populacional de cigarrinhas (Cicadellidae:
Cicadellinae) potenciais vetoras de Xylella fastidiosa associadas à cultura de citros no Vale do Caí, RS. Caderno de Pesquisa, Série Biologia 25: 54-64.

Freitag JH (1951) Host range of Pierce's disease virus of grapes as determined by insect transmission. Phytopathology 41: 920934.

Fundecitrus (1999) Descobertos mais seis vetores de CVC. Revista Fundecitrus 94: 8-9.

Fundecitrus (2007) Manual Técnico de CVC. Fundecitrus, Bebedouro, SP, Brazil.

Gravena S, Lopes JRS, Paiva PEB, Yamamoto PT \& Roberto SR (1998) Os vetores da Xylella fastidiosa. Clorose Variegada dos Citros (ed. by LC Donadio \& CS Moreira), pp. 37-53. Fundecitrus, Bebedouro, SP, Brazil.

Haelterman RM, Tolocka PA, Roca ME, Guzmán FA, Fernández FD \& Otero ML (2015) First presumptive diagnosis of Xylella fastidiosa causing olive scorch in Argentina. Journal of Plant Pathology 97: 393.

Hill BL \& Purcell AH (1995) Acquisition and retention of Xylella fastidiosa by an efficient vector. Phytopathology 85: 209-212.

Hopkins DL \& Purcell AH (2002) Xylella fastidiosa: cause of Pierce's disease of grapevine and others emergent diseases. Plant Disease 86: 1056-1066.

Janse JD \& Obradovic A (2010) Xylella fastidiosa: its biology, diagnosis, control and risks. Journal of Plant Pathology 92: S1.35-S1.48.

Krugner R, Lopes MTV, Santos JS, Beretta MJG \& Lopes JRS (2000) Transmission efficiency of Xylella fastidiosa to citrus by sharpshooters and identification of two new vector species. Proceedings of 14th International Organization of Citrus Virologists Conference, p. 423.

Krugner R, Sisterson MS, Chen J, Stenger DC \& Johnson MW (2014) Evaluation of olive as a host of Xylella fastidiosa and associated sharpshooter vectors. Plant Disease 98: 11861193.

Lawson PB (1931) The genus Xerophloea in North America (Homoptera, Cicadellidae). Pan-Pacific Entomologist 7: 159169.

Li WB, Pria WD, Teixeira DC Jr, Miranda VS, Ayres AJ et al. (2001) Coffee Leaf Scorch caused by a strain of Xylella fastidiosa from citrus. Plant Disease 85: 501-505.

Linnavuori R (1959) Revision of the Neotropical Deltocephalinae and some related subfamilies (Homoptera). Annales Botanici Societatis Zoologicæ-Botanicæ Fennicæ 'Vanamo' 20: 1-370.

Loconsole G, Potere O, Boscia D, Altamura G, Djelouah K et al. (2014) Detection of Xylella fastidiosa in olive trees by molecular and serological methods. Journal of Plant Pathology 96: 7-14.

Lopes JRS (1996) Mecanismo de transmissão de Xylella fastidiosa por cigarrinhas. Laranja 17: 79-92.

Lopes JRS (1999) Estudos com vectores de Xylella fastidiosa e implicações no manejo da clorose variegada dos citros. Laranja 20: 329-344.

Marucci RC, Lopes JRS, Vendramim JD \& Corrente JE (2004) Feeding site preference of Dilobopterus costalimai Young and Oncometopia facialis (Signoret) (Hemiptera: Cicadellidae) on citrus plants. Neotropical Entomology 33: 759-768. 
Marucci RC, Lopes JRS \& Cavichioli RR (2008) Transmission efficiency of Xylella fastidiosa by sharpshooters (Hemiptera: Cicadellidae) in coffee and citrus. Journal of Economic Entomology 101: 1114-1121.

Minsavage GV, Hopkins DL, Leite RM \& Stall RE (1993) Comparison of PCR amplification of DNA and ELISA for the detection of Xylella fastidiosa in plant extracts. Phytopathology 83: 1399.

Minsavage GV, Thompson CM, Hopkins DL, Leite RM \& Stall RE (1994) Development of a polymerase chain reaction protocol for detection of Xylella fastidiosa in plant tissue. Phytopathology 84: 456-461.

Mizell RF III, Andersen PC, Tipping C \& Brodbeck B (2003) Xylella fastidiosa Diseases and their Leafhopper Vectors. University of Florida, IFAS Extension Document ENY-683, UF-Entomology and Nematology Department, Gainesville, FL, USA.

Nault L \& Ammar E (1989) Leafhopper and planthopper transmission of plant viruses. Annual Review of Entomology 34: 503-529.

Nielson MW (1968) The leafhopper vectors of phytopatogenic virus (Homop.: Cicadellidae). Technical Bulletin of the USDA 1382: $1-368$.

Paradell S \& Remes Lenicov AMM (2005) Redescription of Clorindaia hecaloides Linnavuori, 1975 and distribution in the Argentina Republic (Hemiptera, Cicadellidae). Bulletin de la Societé Entomologique de France 10: 133-136.

Paradell SL, Virla EG, Logarzo GA \& Dellapé G (2012) Proconiini sharpshooters of Argentina, with notes on its distribution, host plants, and natural enemies. Journal of Insect Science 12: 1-17.

Parra JRP, Oliveira HN \& Pinto AS (2003) Pragas e Insetos Benéficos com Citros. FEALQ, Piracicaba, SP, Brazil.

Pooler MR \& Hartung JS (1995) Specific PCR detection and identification of Xylella fastidiosa causing citrus variegated chlorosis. Current Microbiology 31: 377-381.

Powell G \& Hardie J (2002) Xylem ingestion by winged aphids. Entomologia Experimentalis et Applicata 104: 103-108.

Purcell AH (1975) Role of the bluegreen sharpshooter, Hordnia circellata, in the epidemiology of Pierce's disease of grapevines. Environmental Entomology 4: 745-752.

Purcell AH (1981) Vector preference and inoculation efficiency as components of resistance to Pierce's disease in European grape Vitis vinifera cultivars. Phytopathology 71: 429-435.

Purcell AH (1985) The ecology of bacterial and mycoplasma plant diseases spread by leafhoppers and planthoppers. The Leafhoppers and Planthoppers (ed. by LR Nault \& JG Rodriguez), pp. 351-380. J Wiley \& Sons, New York, NY, USA.

Purcell AH (1994) Cigarrinhas na cultura de citros. Seminário Internacional de Citros - MIP 3, Campinas, pp. 195-209. Fundação Cargill, Brooklin, SP, Brazil.

Purcell AH \& Finlay AH (1979) Evidence for noncirculative transmission of Pierce's disease bacterium by sharpshooter leafhoppers. Phytopathology 69: 393-395.
Qin X, Miranda VS, Machado MA, Lemos EGM \& Hartung JS (2001) An evaluation of the genetic diversity of Xylella fastidiosa isolated from diseased citrus and coffee in São Paulo, Brazil. Phytopathology 91: 599-605.

Redak R, Purcell A, Lopes JRS, Blua M, Mizell RF \& Andersen PC (2004) The biology of xylem fluid-feeding insect vectors of Xylella fastidiosa and their relation to disease epidemiology. Annual Review of Entomology 49: 243-270.

Remes Lenicov AMM (1982) Aportes al conocimiento de los Agalliinae argentinos. (Homoptera-Cicadellidae). Neotropica 28: 125-138.

Remes Lenicov AMM, Virla EG \& Manca ME (1998) Difusión de Tapajosa rubromarginata (Homoptera: Cicadellidae) sobre cultivos cerealeros de la Argentina. Revista de la Sociedad Entomológica Argentina 57: 18.

Saponari M, Boscia D, Nigro F \& Martelli GP (2013) Identification of DNA sequences related to Xylella fastidiosa in oleander, almond and olive trees exhibiting leaf scorch symptoms in Apulia (Southern Italy). Journal of Plant Pathology 95: 668.

Saponari M, Loconsole G, Cornara D, Yokomi RK, Stradis A et al. (2014) Infectivity and transmission of Xylella fastidiosa by Philaenus spumarius (Hemiptera: Aphrophoridae) in Apulia, Italy. Journal of Economic Entomology 107: 13161319.

Schaad NW, Postnikova E, Lacy G, Fatmi M \& Chang CJ (2004) Xylella fastidiosa subspecies: X. fastidiosa subsp. piercei, subsp. nov., X. fastidiosa subsp. multiplex subsp. nov., and X. fastidiosa subsp. pauca subsp. nov. Systematic and Applied Microbiology 27: 290-300, 763.

Schuenzel EL, Scally M, Stouthamer R \& Nunney L (2005) The multi-gene phylogenetic study of clonal diversity and divergence in North American strains of the plant pathogen Xylella fastidiosa. Applied and Environmental Microbiology 71: 38323839.

Silveira Neto S, Nakano O, Barbin D \& Villa Nova NA (1976) Manual de Ecologia dos Insetos. Agronômica Ceres, Piracicaba, SP, Brazil.

Silveira Neto S, Monteiro RC, Zucchi RA \& Moraes RCB (1995) Uso da análise faunística de insetos na avaliação do impacto ambiental. Scientia Agricola 52: 9-15.

Sokal RR \& Rohlf FJ (1999) Introducción a la Bioestadística. Reverté, Barcelona, Spain.

Stancanelli G, Almeida R, Bosco D, Caffier D, Czwienczek E et al. (2015) Assessing the risk posed to plant health by Xylella fastidiosa in the European Union. CIHEAM International Centre for Advanced Mediterranean Agronomic Studies, Watch Letter 33: 1-8.

Yamamoto PT \& Lopes JRS (2004) Cigarrinhas na proliferação da clorose variegada dos citros. Visão Agrícola 2: 60-63.

Yamamoto PT, Roberto SR, Praia-Júnior WD, Felippe MR, Miranda VS et al. (2000) Transmissao de Xylella fastidiosa pelas cigarrinhas Homalodisca ignorata, Acrogonia virescens e Molomea cincta (Hemiptera: Cicadellidae) em plantas cítricas. Summa Phytopathologica 26: 128. 
Yamamoto PT, Felippe RM, Caetano AC, Sanches AL \& Lopes JRS (2007) First report of Fingeriana dubia Cavichioli transmitting Xylella fastidiosa to citrus. Fitopatologia Brasileira 32: 266.

Young DA (1952) A reclassification of western hemisphere Typhlocybinae (Homoptera, Cicadellidae). University of Kansas Science Bulletin 35: 1-217.

Young DA (1968) Taxonomic study of the Cicadellinae (Homoptera: Cicadellidae), part 1, Proconiini. Bulletin of the United States National Museum 261: 1-287.
Young DA (1977) Taxonomic study of the Cicadellinae (Homoptera: Cicadellidae), part 2, New World Cicadellini and the genus Cicadella. Technical Bulletin of the North Carolina Agricultural Experiment Station 239: 1-1135.

Zar H (1984) Biostatistical Analysis. Prentice Hall, Englewood Cliffs, NJ, USA.

Zhang J, Lashomb J, Gould A \& Hamilton G (2011) Cicadomorpha insects associated with bacterial leaf scorch infected oak in central New Jersey. Environmental Entomology 40: 11311143. 